\title{
Enriched Acoustic Environment after Noise Trauma Reduces Hearing Loss and Prevents Cortical Map Reorganization
}

\author{
Arnaud J. Noreña ${ }^{1}$ and Jos J. Eggermont ${ }^{2}$ \\ ${ }^{1}$ Neurosciences and Sensory Systems Laboratory, Unité Mixte de Recherche Centre National de la Recherche Scientifique 5020, Université Claude Bernard, \\ 69366 Lyon, cedex 7, France, and 2Department of Physiology and Biophysics, Department of Psychology, University of Calgary, Calgary, Alberta, Canada, \\ T2N $1 \mathrm{~N} 4$
}

Exposure to sound of sufficient duration and level causes permanent damage to the peripheral auditory system, which results in the reorganization of the cortical tonotopic map. The changes are such that neurons with pre-exposure tuning to frequencies in the hearing loss range now become tuned to frequencies near the near-normal lower boundary of the hearing loss range, which thus becomes over represented. However, cats exposed to a traumatizing noise and immediately thereafter placed for a few weeks in an enriched acoustic environment presented a much-restricted hearing loss compared with similarly exposed cats that were placed for the same time in a quiet environment. The enriched environment spectrally matched the expected hearing loss range and was $\sim 40 \mathrm{~dB}$ above the level of the expected hearing loss. The hearing loss in the quiet environment-reared cats ranged from 6 to $32 \mathrm{kHz}$ with the largest loss (on average, 40 $\mathrm{dB}$ ) ranging from 24 to $32 \mathrm{kHz}$. In contrast, the hearing loss in the enriched-environment cats was restricted to $6-8 \mathrm{kHz}$ at a level of, on average, $35 \mathrm{~dB}$ and with $16-32 \mathrm{kHz}$ having normal thresholds. Despite the remaining hearing loss for the enriched-environment cats in the $6-8 \mathrm{kHz}$ range, plastic tonotopic map changes in primary auditory cortex could no longer be demonstrated, suggesting that the enriched acoustic environment prevents this reorganization. This finding has implications for the treatment of hearing disorders, such as tinnitus, that have been linked to cortical tonotopic map reorganization.

Key words: plasticity; hearing loss; enriched acoustic environment; tinnitus; auditory; mapping

\section{Introduction}

Hearing loss is highly prevalent in our noisy society and seriously affects the quality of life through impairment of speech understanding (Moore, 1995) and the emergence of phantom sounds or tinnitus (Loeb and Smith, 1967; Chermack and Dengerink, 1987; Temmel et al., 1999; Noreña et al., 2002; Noreña and Eggermont, 2003). Acoustic trauma is one of the main causes of hearing impairment. Noise-induced hearing loss presents cochlear (Liberman and Kiang, 1978; Liberman and Dodds, 1987; Liberman, 1987) and auditory nerve fiber damage (Puel, 1995; Puel et al., 1998; Pujol and Puel, 1999). Pharmacological treatments conventionally administrated after a noise trauma are usually inefficient or of little benefit (Duan et al., 2002). Thus, noiseinduced hearing loss is assumed to be permanent. In this context, it is well established that sound conditioning before inducing a noise trauma reduces the amount of hearing loss (Canlon et al., 1988; Niu and Canlon, 2002; Niu et al., 2003). More interestingly, one study found that unilateral removal of middle ear ossicles after an acoustic trauma, resulting in an attenuation of environ-

Received June 8, 2004; revised Nov. 9, 2004; accepted Nov. 30, 2004.

This work was supported by the Alberta Heritage Foundation for Medical Research, the National Sciences and Engineering Research Council, a Canadian Institutes of Health Research-New Emerging Teams grant, the Canadian Language and Literacy Research Network, and the Campbell McLaurin Chair for Hearing Deficiencies.

Correspondence should be addressed to Jos J. Eggermont, Department of Physiology and Biophysics, Department of Psychology, University of Calgary, Calgary, Alberta, Canada, T2N 1N4. E-mail: eggermon@ucalgary.ca.

DOI:10.1523/JNEUROSCI.2226-04.2005

Copyright $\odot 2005$ Society for Neuroscience $\quad$ 0270-6474/05/250699-07\$15.00/0 mental sound of $\sim 50 \mathrm{~dB}$ over a wide frequency band $(250 \mathrm{~Hz}$ to $8 \mathrm{kHz}$ ), is associated with greater hearing loss in that ear compared with that in the opposite ear with intact middle ear (Fukushima et al., 1990). This suggests that acoustic stimulation immediately after a noise trauma might reduce cochlear lesions. The first goal of the present study was to address whether a posttrauma acoustic stimulation might influence the amount of noise-induced hearing loss.

It is known that cochlear damage of sufficient level and extent is followed by reorganization of the cortical tonotopic map (Robertson and Irvine, 1989; Rajan et al., 1993; Eggermont and Komiya, 2000). Namely, neurons with a characteristic frequency (CF) within the frequency band of the hearing loss acquire CFs corresponding to frequencies at the edge of the high-frequency hearing loss. Consequently, this cutoff frequency of the hearing loss becomes "over represented" at a central level (i.e., more neurons than normal are most sensitive to this frequency) (Rajan et al., 1993; Eggermont and Komiya, 2000). It has been proposed that this central reorganization might cause tinnitus or phantom sounds (Rauschecker, 1999; Noreña et al., 2002; Noreña and Eggermont, 2003). Central reorganization is likely triggered by the decrease in spontaneous and driven firing rate in auditory nerve fibers observed after cochlear damage (Rajan et al., 1993; Noreña et al., 2002, 2003; Noreña and Eggermont, 2003). The second goal of the present study was to address whether an enriched acoustic environment within the frequency region of the hearing loss may 
prevent central reorganization by compensating for the decrease in firing rate caused by the hearing loss.

\section{Materials and Methods}

Acoustic trauma and targeted acoustic stimulation. Fourteen (awake) pigmented short-hair, wild-type cats were exposed to a traumatizing noise. Seven of the exposed cats (weighing 2027 g, on average; range, 1290$2950 \mathrm{~g}$ ) were placed in their free-range room without additional acoustic stimulation (group 1), whereas the other seven cats (weighing $3044 \mathrm{~g}$, on average; range, 2280-4000 g) were placed in the same room with an enriched acoustic environment (group 2). One cat from group 2 was not included in additional analysis, because it did not show any hearing loss after the exposure. Cats in group 2 were continuously stimulated after the trauma with a high-frequency multitone complex stimulus. This stimulus was composed of tone-pips of random frequency (16 logarithmically spaced frequencies per octave) between 0.625 and $20 \mathrm{kHz}$, high-pass filtered (cutoff frequency $=8 \mathrm{kHz}$; slope $=24 \mathrm{~dB} /$ octave), and presented at a level of $80 \mathrm{~dB}$ sound pressure level (SPL). The level of $80 \mathrm{~dB}$ SPL was chosen according to two criteria. The level had to be high enough to stimulate the auditory system taking account of the expected hearing loss in the high frequencies ( $40 \mathrm{~dB}$ loss in group 1). Furthermore, the level had to be low enough not to further damage the auditory system, taking into account that the multitone complex stimulus was on $24 \mathrm{~h} / \mathrm{d}$. We chose to stimulate for $24 \mathrm{~h} / \mathrm{d}$ to maximize the effect on the auditory system after the trauma.

Tone-pip onset times per frequency were Poisson distributed (with a dead time of $50 \mathrm{~ms}$, equal to the duration of the tone pips) at a mean rate of $3 \mathrm{~Hz}$ but independently from that at other frequencies (Blake and Merzenich, 2002). The aggregate tone-pip presentation rate for 81 frequencies was thus $240 \mathrm{~Hz}$. The free-range cat room was $1.7 \times 3.45 \mathrm{~m}$ in floor size and $3 \mathrm{~m}$ high, and it was tiled and acoustically reflective. Two speakers (Realistic Minimus 3.5; Radio Shack, Barrie, Ontario, Canada) were attached on each side and just above the door level at the narrow end of the room and received the same input signal. The overall A-weighted linear scale sound level was measured using a Quest Electronics model 1800 (Quest Scientific, North Vancouver, British Columbia, Canada) precision impulse integrating sound level meter at 12 equidistant points at $10 \mathrm{~cm}$ above floor level and was, on average, $80 \mathrm{~dB}$ SPL (range, 76-82 $\mathrm{dB}$ ) with the measuring points against the wall below the speakers showing the lowest level (76 dB/octave).

To introduce a high-frequency hearing loss, cats were exposed in the anechoic room for $2 \mathrm{~h}$ to a one-third octave band of noise centered at 5 $\mathrm{kHz}$. Measured at the cat's head, the noise had a level of $\sim 120 \mathrm{~dB}$ SPL, a level that has previously been shown to introduce $\sim 40 \mathrm{~dB}$ hearing loss (Eggermont and Komiya, 2000). The sound was presented by a highpower amplifier (Samson Servo 240; Samson Technologies, Hicksville, NY) and loudspeaker (120 W; Yorkville Sound, Pickering, Ontario, Canada) placed $50 \mathrm{~cm}$ away from the animal's head. During this exposure, the animals were awake, confined in a small cage, and facing the loudspeaker. Cats in group 1 were exposed once for $2 \mathrm{~h}(n=3)$ or twice for $2 \mathrm{~h}$ ( $n=4 ; 1$ week separating the two exposures), respectively. The induced hearing loss was not significantly different for these two subgroups. Cats in group 2 were exposed once for $4 \mathrm{~h}(n=4)$ or once for $2 \mathrm{~h}(n=2)$. The hearing losses in these two groups were similar and not significantly different at all tested frequencies [the group exposed for $4 \mathrm{~h}$ presented, nevertheless, greater hearing loss, by $\sim 15 \mathrm{~dB}$, measured by auditory brainstem responses (ABRs) and multiunit (MU) activity in primary auditory cortex (AI) at 12 and $16 \mathrm{kHz}$.

Animal preparation. At the time of the recordings, animals were 125 (range, 107-142), 178 (range, 150-198), and 220 (range, 169-255) days of age, on average, in the control group, group 1, and group 2, respectively. Cortical responses in cats, specifically those related to CFthreshold, tonotopic gradient, and frequency tuning, are mature at $100-$ $120 \mathrm{~d}$ (Eggermont, 1996a; Bonham et al., 2004). In the two exposed groups, recordings were made at least $26 \mathrm{~d}$ after the induction of the acoustic trauma. All animals were deeply anesthetized with the administration of $25 \mathrm{mg} / \mathrm{kg}$ ketamine hydrochloride and $20 \mathrm{mg} / \mathrm{kg}$ sodium pentobarbital injected intramuscularly. A mixture of $0.2 \mathrm{ml}$ of acepromazine $(0.25 \mathrm{mg} / \mathrm{ml})$ and $0.8 \mathrm{ml}$ of atropine methyl nitrate $(25 \mathrm{mg} / \mathrm{ml})$ was administered subcutaneously at $\sim 0.25 \mathrm{ml} / \mathrm{kg}$ body weight. The cat was secured with one screw cemented on the head without any other restraint, and there were no pressure points such as ear bars. The wound margins were infused every $2 \mathrm{~h}$ with lidocain, and additional acepromazine/atropine mixture was administered every $2 \mathrm{~h}$. Throughout the experiment, typically every one-half hour, we tested whether the anesthesia level was sufficient to ensure that pinna reflexes induced by touching the tragus were absent. In addition, careful monitoring of the rate of breathing provided additional information. The mean ketamine dose to maintain anesthesia in this set of animals was $7.6 \pm 2.4 \mathrm{mg} / \mathrm{kg}^{\star} \mathrm{h}$ (range, $\left.3.0-12.8 \mathrm{mg} / \mathrm{kg}^{\star} \mathrm{h}\right)$. This dose is nearly twice that $\left(2-4 \mathrm{mg} / \mathrm{kg}^{\star} \mathrm{h}\right.$ ) used by Escabi and Schreiner (2002) and Escabi et al. (2003). That dose was reported to be sufficient to keep the cat in a state of areflexive anesthesia. The care and the use of animals reported in this study was approved (BI 2001-021) and reviewed on a yearly basis by the Life and Environmental Sciences Animal Care Committee of the University of Calgary. All animals were maintained and handled according to the guidelines set by the Canadian Council of Animal Care.

The ear contralateral to the speaker (ipsilateral to the cortex recorded from) was filled with an ear mold substance (Dur-A-Sil; Insta-Mold Products, Starkey Labs, Mississauga, Ontario, Canada). In humans, this produces a threshold shift of $\sim 30 \mathrm{~dB}$ in the frequency range above $1 \mathrm{kHz}$. In one normal hearing cat (not included in the present series), we found that ear molds inserted attenuated, on average, $32 \mathrm{~dB}$ in the frequency range above $600 \mathrm{~Hz}$.

Peripheral threshold estimation. Peripheral hearing sensitivity was determined from ABR thresholds in an anechoic room. The use of ABR in cats has been validated by comparison of the wave 4 threshold (the largest peak in the cat ABR) to that of auditory nerve fibers in normal animals and those with noise-induced hearing loss by Ngan and May (2001). They found that the ABR thresholds "maintained a systematic relationship to auditory nerve fiber thresholds and therefore have the potential to be used as a functional assay of cochlear pathology" (Ngan and May, 2001). We used the component in the ABR that was detectable at the lowest sound levels, and this was nearly always wave 4, putatively representing synchronized input to the inferior colliculus. Tone pips with frequencies of $3,4,6,8,12,16,24$, and $32 \mathrm{kHz}$ were presented (monaurally, see above) at a rate of $10 / \mathrm{s}$ in an anechoic room from a speaker placed $45^{\circ}$ from the midline and at $55 \mathrm{~cm}$ distance from the cat's head. The half-octave distance is sufficient to characterize a hearing loss of the size we induce; even if a notch-like hearing loss is the result, this typically has a bandwidth of at least one-half octave (Seki and Eggermont, 2002). Calibration and monitoring of the sound field was done using a Brüel and Kjäer (type 4134) microphone placed above the animal's head and facing the loud speaker. ABRs were recorded, with needle electrodes in ipsilateral and contralateral muscles covering both mastoids, in response to gamma function-shaped tone pips with an effective (50\% from peak) duration of $15 \mathrm{~ms}$. The signals were amplified between 300 and $3000 \mathrm{~Hz}$ using a DAM 500 (World Precision Instruments, Sarasota, FL) differential amplifier and averaged with a Brüel and Kjær (type 2034; Brüel and Kjær, Norcross, GA) dual signal analyzer in the signal enhancement mode. Artifact rejection and local lidocaine infusion were used to avoid contamination of the ABR by muscle action potentials. At high intensity levels, 20-50 averages sufficed, but at near threshold values, 200-300 averages were obtained and repeated once. Level was decreased (step size, $10 \mathrm{~dB}$ ) for as long responses were reproducible. Threshold was defined as the highest sound level that yielded a nonreproducible response $+5 \mathrm{~dB}$ SPL. Hearing loss was estimated by comparing ABR thresholds obtained in the cats of the present study with reference ABR thresholds based on data collected in 13 normal hearing cats that were not further part of this study.

Acoustic stimulus presentation. Stimuli were generated in MatLab (MathWorks, Natick, MA) and transferred to the digital signal processing boards of a TDT-2 (Tucker Davis Technologies, Gainesville, FL) sound delivery system. Acoustic stimuli were presented in an anechoic room from a speaker system [Fostex RM765 in combination with a Realistic Super-Tweeter that produced a flat spectrum $( \pm 5 \mathrm{~dB})$ up to 40 $\mathrm{kHz}$ measured at the cat's head] placed $\sim 30^{\circ}$ from the midline into the contralateral field, $\sim 50 \mathrm{~cm}$ from the cat's left ear. Calibration and mon- 
itoring of the sound field was accomplished with a condenser microphone (Brüel and Kjær 4134) placed above the animal's head, facing the speaker and a measuring amplifier (Brüel and Kjær 2636).

The characteristic frequency and tuning properties of individual neurons were determined using the same gamma tone pips (Eggermont, $1996 \mathrm{~b}$ ) as used for ABR recordings. These tone pips with a half-peak amplitude duration of $15 \mathrm{~ms}$ and a gamma function-shaped envelope that was truncated at $50 \mathrm{~ms}$ were presented at a rate of $1 / \mathrm{s}$ in a pseudorandom frequency order at fixed intensity level. The stimulus ensemble consisted of five identical sequences of 81 tone pips covering five octaves (with a tone separation of $1 / 16$ octave), from $625 \mathrm{~Hz}$ to $20 \mathrm{kHz}$ or from $1250 \mathrm{~Hz}$ to $40 \mathrm{kHz}$. The intensity series generally covered the range from 75 or $65 \mathrm{~dB}$ SPL to threshold in $10 \mathrm{~dB}$ steps. Another (faster) procedure was used to characterize frequency-tuning properties of neurons. Here, 27 tone frequencies covering five octaves were presented at eight different intensities (i.e., mainly between 5 and $75 \mathrm{~dB}$ SPL by $10 \mathrm{~dB}$ steps), and each intensity-frequency combination was repeated five times. The presentation rate was $4 / \mathrm{s}$. This method provided very similar results (tuning curves) compared with those obtained with the first method.

Recording and spike separation procedure. Two arrays of eight electrodes (Frederick Haer Company, Bowdoinham, ME), each with impedances between 1 and $2 \mathrm{M} \Omega$, were used. The electrodes were arranged in a $4 \times 2$ configuration with interelectrode distance within rows and columns equal to $0.5 \mathrm{~mm}$. Each electrode array was oriented such that all electrodes were touching the cortical surface and then were manually and independently advanced using a Narishige (Tokyo, Japan) M101 hydraulic microdrive (one drive for each array). The signals were amplified 10,000 times using a Frederick Haer Company HiZx8 set of amplifiers with filter cutoff frequencies set at $300 \mathrm{~Hz}$ and $5 \mathrm{kHz}$. The amplified signals were processed by a DataWave multichannel data acquisition system. Spike sorting was done off-line using a semiautomated procedure based on principal component analysis (Eggermont, 1990) implemented in MatLab. The spike times and waveforms were stored. The multiunit data presented in this paper represent only well-separated single units that, because of their regular spike wave form, likely are dominantly from pyramidal cells (Eggermont, 1996b).

Data analysis. To assess frequency-tuning properties, the peak number of action potentials in the poststimulus time histogram ( $5 \mathrm{~ms}$ bins) over the first $100 \mathrm{~ms}$ after gamma-tone presentation was estimated (action potentials were not summed over the entire time window). The peak counts for three adjacent frequencies were combined (for the first method) to reduce variability and divided by number of stimuli and presented as a peak firing rate per stimulus. This resulted in 27 frequency bins covering five octaves so that the final frequency resolution for determining the $\mathrm{CF}$ was $\sim 0.2$ octaves. The results were calculated per stimulus intensity and combined into an intensity-frequency-rate profile from which tuning curves, rate-intensity functions, and iso-intensity rate-frequency contours could be derived (Eggermont, 1996b) using routines implemented in MatLab. The frequency-tuning curve was defined for a firing rate at $25 \%$ of the maximum peak firing rate (defined across all frequency intensities tested). This was $\sim 10-20 \%$ above the background firing rate, but because the latter was dependent on the level of stimulus-induced suppression, the tuning curve criterion based on a percentage of peak firing rate was preferred over that based on increase over background activity. For the fast method, the same procedure was used. The threshold was determined as the lowest intensity that produced visible responses at $25 \%$ of the peak rate. However, when the tuning curve derived by this method was "noisy" or unclear (for bursting neurons for instance), the threshold was determined as the lowest intensity that produced visible time-locked responses to the tone pip as judged from the dot-raster display.

\section{Results}

We present data from 19 cats. Fourteen cats were exposed to a traumatizing sound. Immediately after the exposure, seven cats (group 2) were placed into a targeted acoustic environment where they were continuously stimulated $(24 \mathrm{~h} / \mathrm{d})$ with a highfrequency multitone stimulus for at least $35 \mathrm{~d}$ (see Materials and

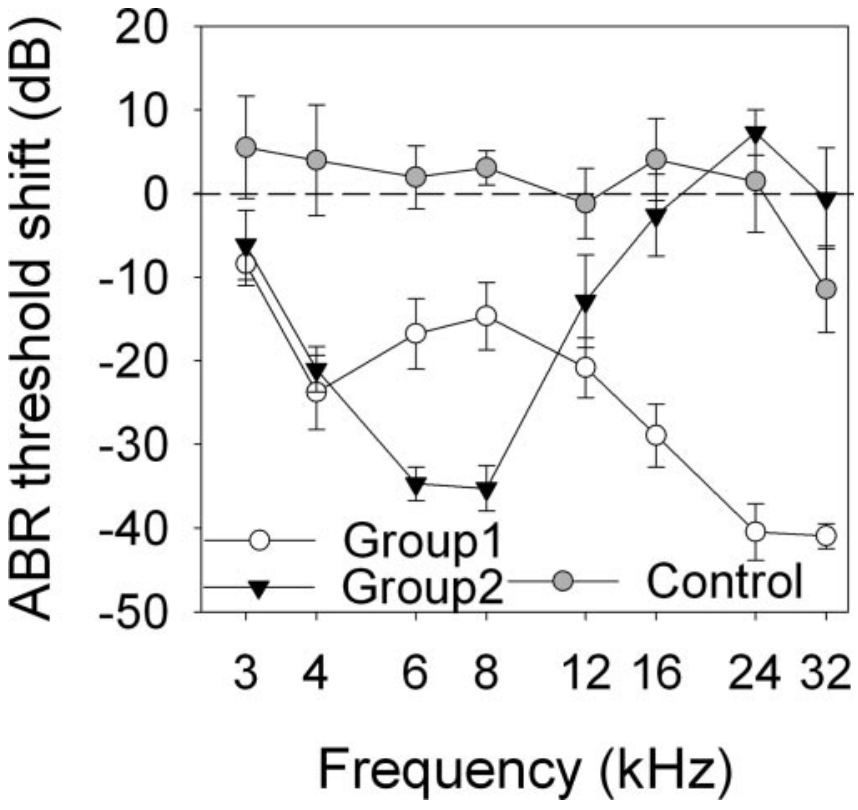

Figure 1. Averaged ABR threshold shifts across the three groups. The threshold shifts in group 1 cats, group 2 cats, and control cats, represented by open circles, filled triangles, and gray filled circles, respectively. A negative shift is considered equal to the amount of hearing loss. Vertical bars indicate the SEM.

Methods). One cat did not show a hearing loss at the time of recording and was not included in the data presented here. The seven other exposed cats (group 1) were placed in the same freerange room without additional sound stimulation for at least $26 \mathrm{~d}$. The six control cats were not noise-exposed and received no additional sound stimulation on top of the normal sounds in the cat room.

\section{Hearing loss}

Peripheral hearing loss was estimated by comparing ABR thresholds to those obtained in a reference group of normal-hearing cats (see Materials and Methods). Figure 1 shows the average difference between the ABR thresholds in the three groups of cats in the present study and in the reference group. A negative difference was considered equal to the amount of hearing loss. The ABR thresholds in each group were statistically compared with the reference group using a one-sample $t$ test; Bonferroni's correction was used to correct for multiple comparisons (eight frequencies; $p<0.00625$ was considered significant). The control group did not show any significant deviation from the reference cats except at the highest frequency tested (one-sample $t$ test, 32 $\left.\mathrm{kHz} ; t_{(5)}=-5.42 ; p=0.0029\right)$. However, this hearing loss was small ( $11 \mathrm{~dB}$, on average). In contrast, all cats exposed to the traumatizing sound presented a hearing loss. Interestingly, the pattern of this hearing loss was very different for the two groups. Group 1 cats (no additional sound stimulation after the trauma) presented a mild hearing loss in the middle-frequency range $\left(\sim 20 \mathrm{~dB}\right.$ at $\left.4 \mathrm{kHz} ; t_{(6)}=-5.33 ; p=0.0018\right)$ and a moderate hearing loss in the high-frequency range $(\sim 30-40 \mathrm{~dB}$ in the frequency band, $16-32 \mathrm{kHz} ;-27.55<t_{(6)}<-5.80 ; 0.0001<$ $p<0.0012)$. In contrast, group 2 cats showed a significant hearing loss in the middle-frequency range $(\sim 20$ and $35 \mathrm{~dB}$ at $4 \mathrm{kHz}$ and $6-8 \mathrm{kHz}$, respectively; $-13<t_{(5)}<-7.75 ; 0.0001<p<$ $0.0006)$, and $A B R$ thresholds above $8 \mathrm{kHz}$ were in the normal range $( \pm 10 \mathrm{~dB})$. This decrease in noise-induced hearing loss in group 2 compared with that in group 1 suggests that an enriched 


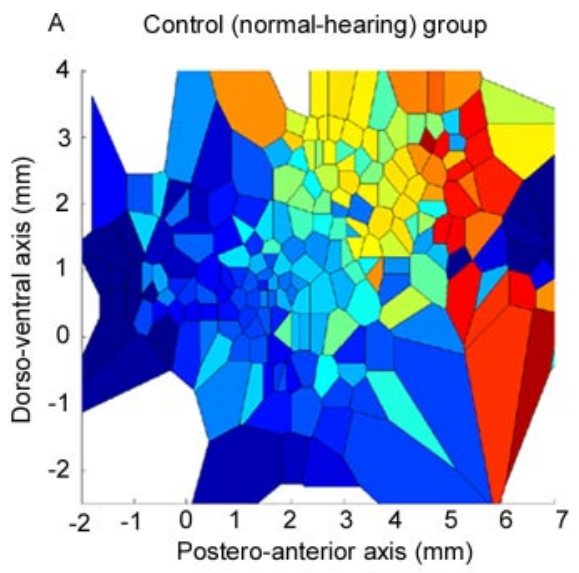

B $\quad$ Group1

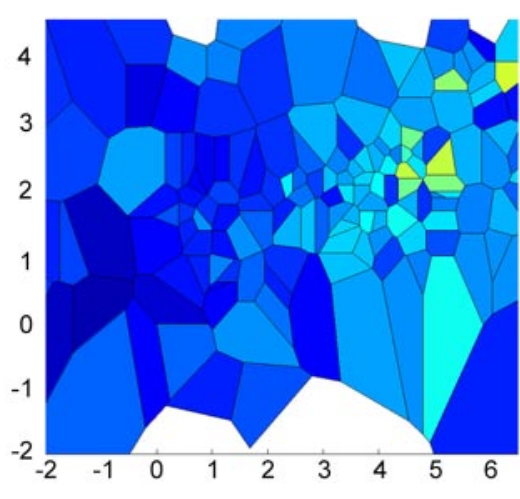

C Group2

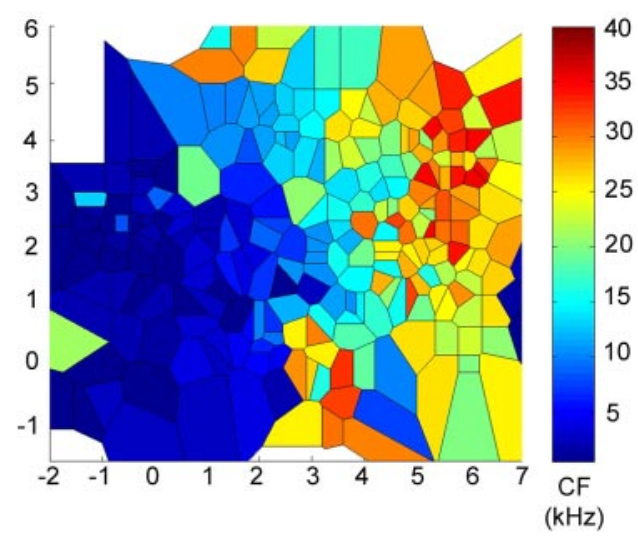

Figure 2. Compound (F maps in Al in control cats $(A)$, group 1 cats $(B)$, and group 2 cats $(C)$. The center of each polygon, constructed using the tessellation method (MatLab), corresponds to the coordinates of a recording site in auditory cortex along the anteroposterior axis (abscises) and the ventrodorsal axis (ordinates). The tip of the posterior ecto-Sylvian sulcus was taken as the $(0,0)$ coordinate. The CF is represented by color; cold colors (blue-like) represent low CF, and hot colors (red-like) represent high CF as indicated by the color bar.

acoustic environment after the noise exposure limited the range of hearing loss as measured several weeks after the trauma.

\section{Cortical tonotopic maps}

The differing effects of the acoustic trauma, clearly dependent on the follow-up stimulation, were also evident in primary auditory cortex where multiple single-unit spike trains were recorded in the middle layers (III-IV). The receptive fields of neurons were obtained simultaneously from up to 16 recording sites in AI (see Materials and Methods). The maps of the CFs obtained at each recording site across all cats in each group are shown in Figure $2 A-C$. The reference point (0.0) was taken as the tip of the posterior ectosylvian sulcus (PES). The horizontal axis is parallel to the posterior-caudal axis of the animal, and the vertical axis consequently runs ventromedial. Although we acknowledge that the primary auditory cortex does not consistently occupy the same region with reference to anatomical landmarks, comparison of group data mapped onto a single anatomical map will reveal any substantial changes in tonotopic maps. The majority of the recording sites were located in AI; however, some recordings were from cortical areas outside AI. For instance, the low-CF neurons in the anterior region of the auditory cortex are certainly located in the anterior auditory field (Fig. $2 A, C$ ). Only recordings from AI (i.e., between boundaries where reversals of the tonotopic gradient occurred) were used for statistical analysis. The boundary between $\mathrm{AI}$ and the anterior auditory field is usually found between $\sim 5$ and $7 \mathrm{~mm}$ from the tip of the posterior ectosylvian sulcus. The CF map obtained in control cats illustrates the cortical tonotopic organization of AI (Fig. 2 A). The CF smoothly increases along the anteroposterior axis up to the anterior auditory field (Merzenich et al., 1973). In contrast, group 1 cats showed a modified tonotopic organization (Fig. 2 B) (i.e., CFs above $30 \mathrm{kHz}$ are no longer present, and those above $20 \mathrm{kHz}$ are severely under represented). Instead, neurons in the anterior part of AI, those that were normally sensitive to high frequencies (Fig. $2 \mathrm{~A}$ ), are now sensitive to a narrow frequency range corresponding to the low-
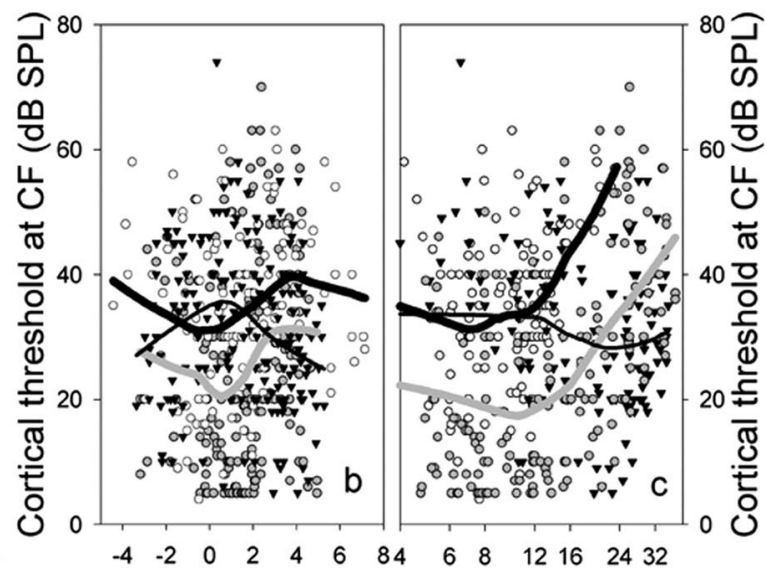

\section{Distance from $8 \mathrm{kHz}$ location $(\mathrm{mm})$}

Figure 3. CF of Al neurons according to the location of the recording site along the anteroposterior axis relative to the $\mathrm{CF}=8$ open circles, filled triangles, and filled gray circles, respectively. Lines represent locally weighted regression curves (solid gray lines, control; thin black lines, group 2; thick black lines, group 1).

frequency boundary of maximum hearing loss (i.e., $\sim 15 \mathrm{kHz}$ ) (Fig. 1). Approximately one-half of the AI tonotopic map is now dedicated to the cutoff frequency of the hearing loss; for that reason, one calls this frequency over represented. This result illustrates the wellknown cortical tonotopic reorganization after a hearing loss (Rajan et al., 1993; Eggermont and Komiya, 2000). Finally, group 2 cats, despite having a hearing loss in the midfrequency range, show an apparent normal tonotopic organization (Fig. 2C). Namely, as already observed in the control group, the $\mathrm{CF}$ smoothly increases along the anteroposterior axis.

Figure $3 a$ shows the CF plotted as a function of the distance with the $\mathrm{CF}=8 \mathrm{kHz}$ location in the anteroposterior direction (Fig. 2, horizontal axis). Because not every cat had an actual $\mathrm{CF}=$ $8 \mathrm{kHz}$ recording site, for each cat, a linear regression line was calculated ( $\mathrm{CF}$ being the dependent variable, and the recording location along the anteroposterior axis being the independent variable). We then derived a theoretical location for $\mathrm{CF}=8 \mathrm{kHz}$ for each cat from the regression lines. This location always corresponded closely to an actual measuring point with $\mathrm{CF}$ at $\sim 8 \mathrm{kHz}$ 
when present. This procedure normalizes the variability in the tonotopic map across cats observed when using anatomical landmarks such as PES (compare Fig. 2). Figure $3 a$ illustrates that the tonotopic organization is similar in control cats and group 2 cats and shows the modified organization of the tonotopic map in group 1. All cats in group 2 and all cats in group 1 showed the same reorganization regardless of noise-exposure time differences. Locally weighted regression curves are drawn in; they are completely overlapping for the control group and group 2. Linear regression lines with 99\% confidence level lines were also calculated for those recordings in $\mathrm{AI}$ with anteroposterior distance above $0 \mathrm{~mm}$ with respect to $\mathrm{CF}=8 \mathrm{kHz}$ but are not shown in Figure $3 a$. Data points in the $99 \%$ confidence interval of these regression lines for group 2 and the control group were essentially overlapping, indicating once more that group 2 cats have a normal tonotopic map. In contrast, data from group 1 did not show any overlap with those of the two latter groups above $3 \mathrm{~mm}$ distance from the tip of the PES. Thus, the anterior part of AI (above $3 \mathrm{~mm}$ anteroposterior distance from the PES) in this group is reorganized. Average CF thresholds were lowest around the $\mathrm{CF}=8 \mathrm{kHz}$ point for the control group but were elevated in both the group 1 and group 2 animals corresponding to the hearing loss in this frequency region (Fig. $3 b$ ).

The change in the tonotopic organization in group 1 could have resulted simply from peripheral hearing loss (i.e., responses of the cortical neurons to their residual afferent input) (Kaltenbach et al., 1992). The residual responses are defined as the neural responses left after the removal of the excitatory inputs around the original CF caused by the hearing loss. MU thresholds at CF as a function of the CF (Fig. 3c). When the neuronal responses in AI are "residual responses," the cortical thresholds should be elevated (Kaltenbach et al., 1992; Rajan et al., 1993). In contrast, a nonsignificant elevation of cortical thresholds in neurons with changed CF would argue for a plastic change of neural responses (Rajan et al., 1993; Eggermont and Komiya, 2000). For group 1, the CF thresholds of MUs corresponding to recording sites above $1 \mathrm{~mm}$ from the recording site of the reference frequency (where the tonotopy starts to be different from that of the control group) were not significantly different (unpaired $t$ test) from those corresponding to recording sites below $3 \mathrm{~mm}$ (Fig. $3 b$ ). This result suggests that the cortical reorganization observed in group 1 is not related to residual responses. Instead, cortical reorganization may have been induced by plastic changes (i.e., by a shift of the receptive field toward the cutoff frequency of the hearing loss) (Rajan et al., 1993; Eggermont and Komiya, 2000). However, a few MUs with a CF above $15 \mathrm{kHz}$ (Fig. 3a) appear to have an elevated threshold and thus may reflect residual responses (Fig. $3 c$ ). For group 2, the CF thresholds of MUs corresponding to recording sites below $2 \mathrm{~mm}$ from the recording site of the reference frequency ( $2 \mathrm{~mm}$ corresponds to $\sim 20 \mathrm{kHz}$ frequency at which the ABR thresholds start to be normal in group 2) were significantly elevated compared with those above $2 \mathrm{~mm}\left[t_{(183)}=\right.$ 3.39; $p=0.0009$ ] (Fig. 3b). This suggests that cortical responses in this group are "residual," namely that they reflect their natural peripheral input (Fig. 1) and, therefore, no cortical reorganization occurred in this group.

Did the enriched acoustic environment prevent tonotopic map reorganization in group 2? After all, it is possible that the amount of hearing loss $(\sim 35 \mathrm{~dB}$ at $6-8 \mathrm{kHz})$ was insufficient to induce reorganization (Rajan and Irvine, 1998; Rajan, 2001). To address this question, we compared the tonotopic organization of one cat from group 2 with that of a "group 1-type" cat having a similar hearing loss (Fig. 4a). This group 1-type cat had been

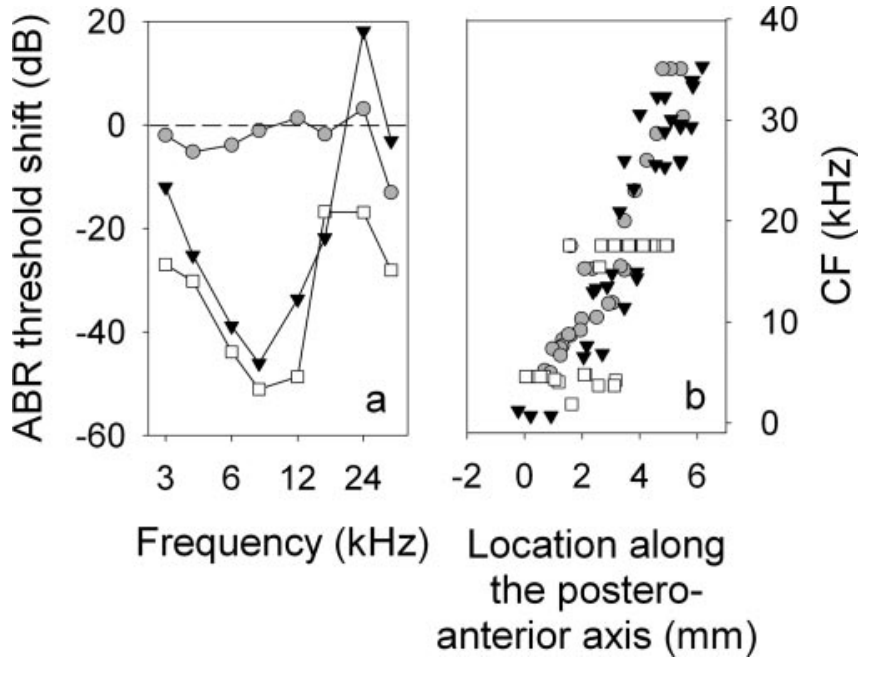

Figure 4. Comparison between two cats having similar hearing loss but different posttrauma exposure and a control (normal hearing) cat. $a, A B R$ threshold shifts. $b, C F$ of Al neurons according to the location of the recording site along the anteroposterior axis. The group 2 cat, group 1-type cat, and the control cat are represented by filled triangles, open squares, and filled gray circles, respectively.

exposed for $2 \mathrm{~h}$ to a $6 \mathrm{kHz}$ pure tone and received no additional stimulation after the trauma (Seki and Eggermont, 2002). The CF-distance map appears normal in the control cat and in the cat from group 2 (Fig. 4b). In contrast, the group 1-type cat presents a strongly modified, reorganized map; namely, only two CFs are represented: one at $\sim 5 \mathrm{kHz}$ and the other at $\sim 17 \mathrm{kHz}$. This result corroborates a previous study where a notched-hearing loss has been shown to induce an overrepresentation of the two cutoff frequencies (Robertson and Irvine, 1989). The fact that the latter cat presented an amount of hearing loss (similar to the averaged hearing loss in group 2) sufficient to induce cortical tonotopic reorganization indicates that it would also have been sufficient for the group 2 cat. Thus, targeted acoustic stimulation following noise trauma may not only have reduced the frequency extent of the hearing loss but also prevented a reorganization of the tonotopic map in group 2. Finally, group 1 shows an enlarged representation of frequencies between 11 and $16 \mathrm{kHz}$ in which the peripheral hearing loss is $\sim 25 \mathrm{~dB}$ (Figs. $2 \mathrm{~B}, 3 a$ ). This result also suggests that cortical reorganization may occur for hearing losses at $\sim 25 \mathrm{~dB}$ as shown previously by Seki and Eggermont (2002). Group 2 cats, with an average hearing loss of $\sim 35 \mathrm{~dB}$ at $6-8 \mathrm{kHz}$, should then have shown a cortical reorganization as well. Thus, an enriched acoustic environment may have prevented the occurrence of tonotopic map reorganization in cortex.

\section{Discussion}

The present study suggests that noise-induced hearing loss can be limited by targeted acoustical stimulation of the exposed animals immediately after the trauma. This reduction in noise-induced hearing loss is maximal in the frequency region above $12 \mathrm{kHz}$. We do not know whether targeted stimulation needs to be continued as long as in the present study. Previous studies (Canlon et al., 1988) have suggested a beneficial effect of acoustic stimulation in protecting the auditory system. Moreover, it has been shown that age-related hearing loss is reduced by life-long acoustic stimulation (Willott and Bross, 2004). However, our study is one of the first to directly show a beneficial effect of targeted acoustic stimulation on peripheral and central aspects of hearing after an acoustic trauma and may have considerable clinical implications. 
A recent study (Niu et al., 2004) showed that low-frequency (1 $\mathrm{kHz}, 81 \mathrm{~dB}$ SPL) sound stimulation applied $2 \mathrm{~h}$ after an acoustic trauma $(2.7 \mathrm{kHz}, 103 \mathrm{~dB}$ SPL, $30 \mathrm{~min})$ for a period of $24 \mathrm{~h}$ reduced peripheral hearing loss as measured $1 \mathrm{~h}$ later, whereas high-frequency $(6.3 \mathrm{kHz}, 78 \mathrm{~dB}$ SPL $)$ stimulation after and acoustic trauma $(5.5 \mathrm{kHz}, 109 \mathrm{~dB}$ SPL, $30 \mathrm{~min})$ had no effect. This latter result contrasts with the findings reported here and may be related to the lower level and the shorter duration of the exposure as well as shorter postexposure stimulation in the Niu et al. (2004) study.

The mechanisms causing the observed partial conservation of hearing are not yet known. Noise-induced hearing loss is related to cochlear damages (Liberman and Kiang, 1978). It has been shown that high-level and long-duration acoustic trauma (at an intensity level of $115 \mathrm{~dB}$ SPL for $2 \mathrm{~h}$ ) induces widespread damage to the stereocilia, mainly those on the inner hair cells and first row of the outer hair cells (Liberman, 1987). Acoustic trauma is also known to induce hair cell and neuron death through formation of reactive oxygen species and free radicals (Duan et al., 2000; Lefebvre et al., 2002). It has been suggested that necrosis and apoptosis are simultaneously involved in hair cell death ( $\mathrm{Hu}$ et al., 2002). The resulting hair cell death and lesions are assumed to be permanent in mammals (Duan et al., 2000). In addition, acoustic trauma causes a degeneration of auditory nerve fiber neurites through excitotoxicity. However, contrary to damage of cochlear hair cells, damage of auditory nerve fibers may be reversible: auditory nerve fiber neurites can regenerate within a few days after excitotoxicity caused by an acoustic trauma. The posttrauma acoustic stimulation may therefore have potentiated the repair at auditory nerve fiber level (Puel, 1995; Puel et al., 1998; Pujol and Puel, 1999) and/or limited the factors causing a hearing impairment at cochlear and/or auditory nerve level (Duan et al., 2000; Lefebvre et al., 2002). A recent report suggests that the recovery after noise-induced hearing loss might also be related partly to the regeneration of hair cell stereocilia (Schneider et al., 2002). The posttrauma acoustic stimulation might have potentiated this regeneration. Finally, it is important to mention that hearing loss in group 2 was not reduced in the frequency band one-half octave above the center frequency of the trauma stimulus $(6-8 \mathrm{kHz})$ (i.e., where hair cell loss was most likely to occur) (Schmiedt, 1984).

It has been suggested that treatments aimed at improving the microcirculation of the cochlea are effective in limiting noiseinduced hearing loss (Duan et al., 2002). Posttrauma stimulation could potentially have improved the microcirculation of the cochlea. By stimulating the intact or still functional inner hair cells, it may also have promoted the release of nerve growth factor, which may have facilitated the repair of damaged auditory nerve fibers (Duan et al., 2000; Oestreicher et al., 2000). In this context, it is important to mention that nerve growth factors are necessary for the survival of auditory nerve fibers in mammals. Indeed, it has been shown that neurotrophin withdrawal was responsible for cell death through an increase production of reactive oxydative species (Dugan et al., 1997; Lefebvre et al., 2002). Additional studies are needed to specify the mechanisms by which acoustic stimulation reduces noise-induced hearing loss.

The present study also suggests that targeted acoustic stimulation after noise-induced hearing loss can prevent cortical tonotopic map reorganization. After a peripheral lesion, it is well known that the neurons with a CF in the frequency region of the hearing loss become sensitive to neighboring regions (i.e., the cutoff frequency of the hearing loss) (Rajan et al., 1993; Eggermont and Komiya, 2000). Here, we did not observe such a sys- tematic change in CF of neurons in the group kept in the enriched acoustic environment (group 2). An explanation could be that the amount of hearing loss was insufficient (Rajan and Irvine, 1998). However, this explanation is unlikely because we showed that similar hearing loss in an animal from a group not subjected to an enriched environment led to tonotopic map reorganization (Fig. 4). Moreover, our results in group 1 suggest that a hearing loss of $20-30 \mathrm{~dB}$ is sufficient to induce tonotopic map reorganization. With an averaged hearing loss of $\sim 35 \mathrm{~dB}$ at $6-8 \mathrm{kHz}$, the group 2 then should have presented reorganized tonotopic maps. Consequently, our results suggest that posttrauma acoustic stimulation may have interfered with the mechanisms causing central reorganization.

It is shown in Figure $3 a$ that some recordings could have been made in the anterior part of the auditory cortex. These recordings might be from anterior auditory field but not necessarily. Indeed, it is possible that because of the type of hearing loss (notch-type) in the two cats that provided these data points and had more hearing loss at $4 \mathrm{kHz}$ than at $12 \mathrm{kHz}$, those neurons originally tuned at $\sim 4 \mathrm{kHz}$ would now be tuned to $12 \mathrm{kHz}$. This would extend the range of values within $\mathrm{AI}$ at least by another millimeter.

In this context, it is important to underline that tonotopic map reorganization is not an "all-or-nothing" phenomenon, namely all neurons do not necessarily show reorganized tuning properties. It is likely that the amount of peripheral damages strongly influences the amount (systematicity) of tonotopic map reorganization (Noreña et al., 2003). It has been shown that the mechanical destruction of the basal part of the cochlea (Rajan et al., 1993) induces a systematic reorganization on all neurons in AI. In contrast, hearing loss caused by an acoustic trauma induces only moderate cochlear damage (Fig. 1). This could explain why some neurons do not show a "reorganized" CF in our study (Fig. $3 a$, group 1). Nevertheless, our data clearly argue for true (plastic) reorganized tuning properties in the majority of neurons. Indeed, in the reorganized region, CFs are lower than expected from a normal map, and these new responses are not likely to reflect residual responses (threshold at CF in the reorganized region are not significantly increased).

Central tonotopic map reorganization is likely initiated by a decrease in spontaneous and driven firing rates in part of the auditory nerve fibers (Rajan et al., 1993). The reduction in spontaneous and driven firing rate after hearing impairment may be induced by damage to inner hair cell stereocilia (Liberman and Dodds, 1984). Indeed, loss or damage of stereocilia might induce a hyperpolarization of inner hair cells. This, in turn, decreases spontaneous firing rate through a decrease in spontaneous release of neurotransmitter (Liberman and Dodds, 1984). This localized decrease in firing rate could thereafter induce a cascade of central changes leading to tonotopic map reorganization (Rajan et al., 1993). It is known that central auditory neurons receive their excitatory inputs from a relative broad frequency band. However, inputs remote from the best frequency of the neuron are usually inhibited or "masked" (Noreña and Eggermont, 2003). At least two different types of inhibitory mechanisms, phasic and tonic, could be involved in the tuning properties of the neurons (Calford et al., 1993; Rajan, 2001; Noreña and Eggermont, 2003; Noreña et al., 2003). The tonic inhibition (driven by spontaneous activity of afferent inputs) is supposed to spread widely across frequency and to be proportional to the amount of spontaneous firing rate of excitatory inputs. When spontaneous firing rates at the auditory nerve level are reduced (caused by hearing loss; see above), this tonic inhibition is also reduced. As a consequence, inputs that were previously inhibited are "un- 
masked" (Calford et al., 1993; Noreña and Eggermont, 2003; Noreña et al., 2003). This release from inhibition can unmask inputs at frequency relatively remote from the hearing loss region. In summary, a decrease in spontaneous firing rate at a peripheral level induces a release from inhibition at central level, causing the emergence of new responses (i.e., reorganization of the tonotopic map) (Calfordet al., 1993; Rajan et al., 1993; Eggermont and Komiya, 2000; Noreña et al., 2003). By stimulating the frequency region corresponding to the hearing loss, we compensated for the decrease in firing rates in the auditory nerve fibers caused by hearing loss and thereby prevented the cascade of central changes (release from inhibition) that would normally lead to cortical tonotopic map reorganization (Noreña et al., 2002; Noreña and Eggermont, 2003).

\section{References}

Blake DT, Merzenich MM (2002) Changes of AI receptive fields with sound density. J Neurophysiol 88:3409-3420.

Bonham BH, Cheung SW, Godey B, Schreiner CE (2004) Spatial organization of frequency response areas and rate/level functions in the developing AI. J Neurophysiol 91:841-854.

Calford MB, Rajan R, Irvine DR (1993) Rapid changes in the frequency tuning of neurons in cat auditory cortex resulting from pure-toneinduced temporary threshold shift. Neuroscience 55:953-964.

Canlon B, Borg E, Flock A (1988) Protection against noise trauma by preexposure to a low level acoustic stimulus. Hear Res 34:197-200.

Chermack GD, Dengerink JE (1987) Characteristics of temporary noiseinduced tinnitus in male and female subjects. Scand Audiol 16:67-73.

Duan M, Agerman K, Ernfors P, Canlon B (2000) Complementary roles of neurotrophin 3 and a $N$-methyl-D-aspartate antagonist in the protection of noise and aminoglycoside-induced ototoxicity. Proc Natl Acad Sci USA 97:7597-7602.

Duan ML, Ulfendahl M, Laurell G, Counter AS, Pyykko I, Borg E, Rosenhall U (2002) Protection and treatment of sensorineural hearing disorders caused by exogenous factors: experimental findings and potential clinical application. Hear Res 169:169-178.

Dugan LL, Creedon DJ, Johnson Jr EM, Holtzman DM (1997) Rapid suppression of free radical formation by nerve growth factor involves the mitogen-activated protein kinase pathway. Proc Natl Acad Sci USA 94:4086-4091.

Eggermont JJ (1990) The correlative brain. Theory and experiment in neural interaction, pp 102-112. Berlin: Springer.

Eggermont JJ (1996a) Differential maturation rates for response parameters in cat primary auditory cortex. Audit Neurosci 2:309-327.

Eggermont JJ (1996b) How homogeneous is cat primary auditory cortex? Evidence from simultaneous single-unit recordings. Audit Neurosci 2:76-96.

Eggermont JJ, Komiya H (2000) Moderate noise trauma in juvenile cats results in profound cortical topographic map changes in adulthood. Hear Res 142:89-101.

Escabi MA, Schreiner CE (2002) Nonlinear spectrotemporal sound analysis by neurons in the auditory midbrain. J Neurosci 22:4114-4131.

Escabi MA, Miller LM, Read HL, Schreiner CE (2003) Naturalistic auditory contrast improves spectrotemporal coding in the cat inferior colliculus. J Neurosci 23:11489-11504.

Fukushima N, White P, Harrison RV (1990) Influence of acoustic deprivation on recovery of hair cells after acoustic trauma. Hear Res 50:107-118.

Hu BH, Henderson D, Nicotera TM (2002) Involvement of apoptosis in progression of cochlear lesion following exposure to intense noise. Hear Res 166:62-71.

Kaltenbach JA, Czaja JM, Kaplan CR (1992) Changes in the tonotopic map of the dorsal cochlear nucleus following induction of cochlear lesions by exposure to intense sound. Hear Res 59:213-223.

Lefebvre PP, Malgrange B, Lallemend F, Staecker H, Moonen G, Van De Water TR (2002) Mechanisms of cell death in the injured auditory system: otoprotective strategies. Audiol Neurootol 7:165-170.

Liberman MC (1987) Chronic ultrastructural changes in acoustic trauma: serial-section reconstruction of stereocilia and cuticular plates.
Liberman MC, Dodds LW (1984) Single-neuron labeling and chronic cochlear pathology. II. Stereocilia damage and alterations of spontaneous discharge rates. Hear Res 16:43-53.

Liberman MC, Dodds LW (1987) Acute ultrastructural changes in acoustic trauma: serial section reconstruction of stereocilia and cuticular plates. Hear Res 26:45-64.

Liberman MC, Kiang NY (1978) Acoustic trauma in cats. Cochlear pathology and auditory nerve activity. Acta Otolaryngol Suppl 358:1-63.

Loeb M, Smith RP (1967) Relation of induced tinnitus to physical characteristics of the inducing stimuli. J Acoust Soc Am 42:453-455.

Merzenich MM, Knight PL, Roth GL (1973) Cochleotopic organization of primary auditory cortex in the cat. Brain Res 63:343-346.

Moore BCJ (1995) Perceptual consequences of cochlear damage. Oxford: Oxford UP.

Ngan EM, May BJ (2001) Relationship between auditory brainstem response and auditory nerve thresholds in cats with hearing loss. Hearing Res 156:44-52.

Niu X, Canlon B (2002) Protective mechanisms of sound conditioning. Adv Otorhinolaryngol 59:96-105.

Niu X, Shao R, Canlon B (2003) Suppression of apoptosis occurs in the cochlea by sound conditioning. NeuroReport 14:1025-1029.

Niu X, Tahera Y, Canlon B (2004) Protection against acoustic trauma by forward and backward sound conditioning. Audiol Neurootol 9:265-273.

Noreña AJ, Eggermont JJ (2003) Changes in spontaneous neural activity immediately after an acoustic trauma: implications for neural correlates of tinnitus. Hear Res 183:137-153.

Noreña AJ, Micheyl C, Chery-Croze S, Collet L (2002) Psychoacoustic characterization of the tinnitus spectrum: implications for the underlying mechanisms of tinnitus. Audiol Neurootol 7:358-369.

Noreña AJ, Tomita M, Eggermont JJ (2003) Neural changes in cat auditory cortex after a transient pure-tone trauma. J Neurophysiol 90:2387-2401.

Oestreicher E, Knipper M, Arnold A, Zenner HP, Felix D (2000) Neurotrophin 3 potentiates glutamatergic responses of IHC afferents in the cochlea in vivo. Eur J Neurosci 12:1584-1590.

Puel J-L (1995) Chemical synaptic transmission in the cochlea. Prog Neurobiol 47:449-476.

Puel JL, Ruel J, Gervais d'Aldin C, Pujol R (1998) Excitotoxicity and repair of cochlear synapses after noise-trauma induced hearing loss. NeuroReport 22:2109-2114.

Pujol R, Puel JL (1999) Excitotoxicity, synaptic repair, and functional recovery in the mammalian cochlea: a review of recent findings. Ann NY Acad Sci 884:249-254.

Rajan R (2001) Plasticity of excitation and inhibition in the receptive field of primary auditory cortical neurons after limited receptor organ damage. Cereb Cortex 11:171-182.

Rajan R, Irvine DR (1998) Neuronal responses across cortical field A1 in plasticity induced by peripheral auditory organ damage. Audiol Neurootol 3:123-144.

Rajan R, Irvine DR, Wise LZ, Heil P (1993) Effect of unilateral partial cochlear lesions in adult cats on the representation of lesioned and unlesioned cochleas in primary auditory cortex. J Comp Neurol 338:17-49.

Rauschecker JP (1999) Auditory cortical plasticity: a comparison with other sensory systems. Trends Neurosci 22:74-80.

Robertson D, Irvine DRF (1989) Plasticity of frequency organization in auditory cortex of guinea pigs with partial unilateral deafness. J Comp Neurol 282:456-471.

Schmiedt RA (1984) Acoustic injury and the physiology of hearing. J Acoust Soc Am 76:1293-1317.

Schneider ME, Belyantseva IA, Azevedo RB, Kachar B (2002) Rapid renewal of auditory hair bundles. Nature 418:837-838.

Seki S, Eggermont JJ (2002) Changes in cat primary auditory cortex after minor-to-moderate pure-tone induced hearing loss. Hear Res 173:172-186.

Temmel AF, Kierner AC, Steurer M, Riedl S, Innitzer J (1999) Hearing loss and tinnitus in acute noise trauma. Wien Klin Wochenschr 111:891-893.

Willott JF, Bross L (2004) Effects of prolonged exposure to an augmented acoustic environment on the auditory system of middle-aged C57BL/6J mice: cochlear and central histology and sex differences. J Comp Neurol 472:358-370. 